angle $\quad\left(\mathrm{M}_{\text {diff }}=4.81, S E=.51, t_{(268)}=9.42, p=.000,95 \% \mathrm{CI}:\right.$ 3.81 - 5.82) than non-ballet students. In contrast, ballet students had lesser mean than non-ballet students in tibiofemoral angle tibiofemoral angle $\left(\mathrm{M}_{\text {diff }}=-1.05, S E=.31, t_{(268)=-3.39}\right.$, $p=.001,95 \%$ CI: $-1.64 \quad-0.44)$, prone rearfoot angle $\left(\mathrm{M}_{\text {diff }}=-8.65, S E=.56, t_{(254.04)=}-16.16, p=.000,95 \% \mathrm{CI}:-9.71\right.$ - -7.60), tibial varum $\left(\mathrm{M}_{\text {diff }}=-2.52, S E=.23, t_{(159.90)}=-10.98\right.$, $p=.000$, 95\% CI: $-2.96--2.06)$, hip anteversion $\left(\mathrm{M}_{\text {diff }}=-\right.$ 11.47, $S E=.72, t_{(156.15)}=-15.87, p=.000,95 \%$ CI: $-12.90--$ $10.04)$, and navicular drop $\left(\mathrm{M}_{\text {diff }}=-4.45, S E=.42, t_{(182.22)=^{-}}\right.$ 10.44, $p=.000,95 \%$ CI: $-5.29--3.61)$.

Conclusions Significant results from the alignment indicate that ballet movement and turn-out position may suggest changes in LEA, therefore, clinicians should consider these aspects while preventing and treating dancer injuries.

\section{INFLUENCE OF LOWER QUARTER Y-BALANCE TESTTM SCREENING PROTOCOL ON DYNAMIC BALANCE OUTCOMES}

${ }^{1}$ Scott Lawrance, ${ }^{1}$ Megan Jacobs, ${ }^{1}$ Emily Boss, ${ }^{1}$ Jennifer Popp, ${ }^{1}$ Larry Leverenz, ${ }^{2}$ Michael Weller. ${ }^{1}$ Purdue University, West Lafayette, IN, USA; ${ }^{2}$ Cedarville University, Cedarville, OH, USA

\subsection{6/bjsports-2021-IOC.236}

Background The Lower Quarter Y-Balance Test ${ }^{\mathrm{TM}}$ (LQYBT) is commonly used to determine risk of injury or readiness for return to sport. However, clinicians conduct the assessment with differing test protocols potentially altering test outcomes. Objective To determine if differences in reach distances, composite score, and limb symmetry exist between LQYBT testing protocols.

Design Prospective cohort study.

Setting Controlled laboratory research.

Participants 48 non-injured participants: 32 females, 16 males $(21.4 \pm 0.3$ years, $170.6 \pm 9.2 \mathrm{~cm}, 72.9 \pm 14.2 \mathrm{~kg})$.

Interventions Participants completed four testing protocols including barefoot with hands fixed at their hips, barefoot with hands free to move, shod with hands fixed at hips, and shod with hands free to move.

Main Outcome Measures Maximum reach distance was recorded for each limb in the anterior, posteromedial, and posterolateral directions. Limb length composite scores and limb symmetry index (LSI) were calculated for each LQYBT assessment.

Results No statistically significant differences were observed in reach distances, composite scores, or LSI between shod and barefoot protocols. Significant differences were observed in reach distances $(p=0.00)$ and in limb length composite scores (right limb $p=0.00$, left limb $p=0.00$ ) between protocols comparing hands fixed at hips and hand free to move, although no differences were observed in LSI between these conditions when participants were shod $(p=0.27)$ or barefoot $(p=0.49)$.

Conclusions No differences were observed when participants wore athletic shoes or were barefoot during assessments. Reach distance and limb composite score differences were present when participants were allowed to move their arms and counterbalance their movement during the LQYBT, although no differences in LSI was observed. Results suggest if LSI is used to make clinical decisions, any LQYBT testing protocol can be used. However, testing protocols could influence clinical decisions if reach distances or composite scores are used to make patient care decisions.

\section{8 SPORT PRE-PARTICIPATION HEALTH EVALUATION IN ELITE ATHLETES FROM A MULTISPORT CLUB: PROPOSAL FOR A PERSONALIZED PROTOCOL}

${ }^{1}$ Ramon Pi, 1,2María Sanz de la Garza, ${ }^{3}$ Gonzalo Grazioli, 1,2Gil Rodas, 'Manel García, ${ }^{1,2}$ Marta Sitges, ${ }^{1,4}$ Francheck Drobnic. ${ }^{1}$ FC Barcelona Medical Department, Barcelona, Spain; ${ }^{2}$ Hospital Clínic, Barcelona, Spain; ${ }^{3}$ Sports Medicine Unit, Consell Catalá de l'Esport, Barcelona, Spain; ${ }^{4}$ Olympic Training Centre, Sant Cugat del Valles, Barcelona, Spain

\subsection{6/bjsports-2021-IOC.237}

Background In 2009 the IOC published a Consensus Statement highlighting the value of periodic health evaluation in elite athletes.

Objective The aim of this study was to evaluate the prevalence of pathological findings, and the preventive value of the method used.

Design Retrospective epidemiological study.

Setting and Participants A total of 2574 elite and professional athletes from Football Club Barcelona (FCB). FCB is a sports club with 13 different sports, 5 professional and 8 amateur. Interventions (or Assessment of Risk Factors) Between 2008 and 2018, a total of 2574 athletes, with range from 12 to 35 years old, were evaluated through a personalized sport preparticipation health evaluation protocol (SPPHE) at the FCB Medical Department.

Main Outcome Measurements The protocol used was the 'Guía de la Revisión Médica del Futbol Club Barcelona'. The SPPHE examination consisted of basic medical information, anthropometric data, physical examination, spirometry, basal 12-lead electrocardiography, submaximal cardiovascular exercise testing, and cardiac echocardiography.

Results In 2574 SPPHE, we recorded 750 medical findings (29.1\%); including 495 (19.2\%) pathological findings, 255 (9.9\%) minor abnormal findings, and 958 (37.2\%) previous sports injuries. Specific treatment was necessary in 6 cardiovascular diseases. We found 3 endocrinological diseases (type 1e Diabetes Mellitus). Periodic follow-up was specified in all cases, although none prevented the athlete from competing.

Conclusions The SPPHE was effective in identifying a wide range of pathologies $(66.3 \%)$ in elite athletes from this multisport club. This allow us to provide fast treatment and implement ad hoc strategic preventive programs. We found no alteration that affected the athlete's development or hindered them in reaching the highest level of competition.

\section{SHOULD THE SIDE-HOP TEST BE REDUCED FROM 40 CM TO 30 CM WHEN USED IN DEVELOPMENTAL ATHLETES?}

${ }^{1,2}$ Isabel Schneider, ${ }^{3}$ Nicolai Kammann, ${ }^{1,3}$ Oliver Haertel. ' German University of Health and Sport, Berlin, Germany; '2Physio-Motion, Groebenzell, Germany; ${ }^{3}$ Leichtathletik Foerderzentrum, Munich, Germany

\subsection{6/bjsports-2021-IOC.238}

Background The side-hop test by Gustavsson et al. (2006) helps with return to play decision making after lower extremity injury.

Objective Determine whether a reduced width of $30 \mathrm{~cm}$ (compared with $40 \mathrm{~cm}$ ) may be more suitable for use in developmental athletes.

Design Cross-sectional study.

Setting School gymnasium. 
Participants 18 developmental athletes, mean age $13.93 \pm$ 1.37 years, (range 11.9 - 16.3 years), all members of an elite athletics project.

Assessment Each participant performed a $40 \mathrm{~cm}$ and a $30 \mathrm{~cm}$ side-hop test for each leg. Athletes were tested during a regular training session, sufficient resting time $(\geq 4 \mathrm{~min})$ granted after each trial. All tests conducted within 10 days. Tests were supervised and evaluated by two coaches, one counting number of jumps during the trial with a customary hand-held mechanical clicker, the other one filming each attempt with a latest generation smartphone high-speed camera. Errors clickcounted on home PC with videos running at half speed and error-rate calculated.

Main Outcome Measurements Comparison of total number of jumps and error-rates for both distances. Evaluation of correlations with time to/from peak height velocity (PHV), height, and chronological age.

Results Lower number of jumps for $40 \mathrm{~cm}(59.33 \pm 8.66)$ vs. $30 \mathrm{~cm}(66.8 \pm 9.91)$, but higher error-rate for $40 \mathrm{~cm}(0.27 \pm$ $0.13)$ compared with $30 \mathrm{~cm}(0.22 \pm 0.12)$. Paired t-tests show significant differences $(p<0.02)$ for both. Based on cohen's $d$, effect is large for comparing number of hops $(\mathrm{d}=0.81)$, but small when comparing error-rates $(\mathrm{d}=0.40)$. Pearsons's correlations of error-rates with age, height, and PHV are stronger for $30 \mathrm{~cm}\left(r_{\text {age }}=-0.664 ; r_{\text {height }}=-0.344 ; r_{P H V}=-0.351\right)$, than for $40 \mathrm{~cm} \quad\left(r_{\text {age }}=-0.537 ; \quad r_{\text {height }}=-0.145 ; \quad r_{P H V}=-0.155\right)$, however, respective effect sizes based on cohen's $\mathrm{q}$ are small $\left(\mathrm{q}_{\text {age }}=0.201 ; \mathrm{q}_{\text {height }}=0.213 ; \mathrm{q}_{\mathrm{PHV}}=0.210\right)$.

Conclusions While results remain statistically inconclusive when comparing $40 \mathrm{~cm}$ and $30 \mathrm{~cm}$ hop-tests in developmental athletes, $30 \mathrm{~cm}$ width may be favourable in a clinical context due to higher number of jumps and lower error rates. Further research is warranted to provide clarity.

\section{THE HIDDEN COST OF WINNING A MEDAL: A NARRATIVE REVIEW ON THE MENTAL HEALTH OF ELITE ATHLETES} ${ }^{1}$ Alina-Maria Smaranda, ${ }^{3}$ Alexandra Vlaic, ${ }^{1,4}$ Adela Caramoci, ${ }^{1,2,4}$ Anca-Mirela lonescu.
${ }^{1}$ Department of Sports Medicine - Carol Davila University of Medicine, Bucharest, Romania;
${ }^{2}$ European Federation of Sports Medicine Associations (EFSMA), Lausanne, Switzerland;
${ }^{3}$ Department of Psychiatry - University Emergency Central Military Hospital Dr. Carol Davila,
Bucharest, Romania; ${ }^{4}$ National Institute of Sports Medicine, Bucharest, Romania

\subsection{6/bjsports-2021-IOC.239}

Background Athletes have started a public conversation about the psychological struggles they are facing. To create a safer psychological environment, the sporting community has the duty to support this movement by increasing awareness, providing educational resources, addressing stigma and encouraging mental health research.

Objective Illustrate an overview of the current literature regarding the mental health of elite athletes.

Design Narrative review

Setting Elite division

Results Physical exercise can reduce the risk of mental illness, nevertheless, at elite levels, the constant physical and psychological stress has a negative impact on athletes' mental health. Consequently, symptoms such as: anhedonia, depressed mood, anxiety, irritability, decreased self-esteem and suicidal ideation can arise and lead to serious psychiatric disorders. A metaanalysis focused on the prevalence of mental health disorders concluded that $34 \%$ of elite athletes suffer from anxiety/ depression and 19\% have reported alcohol misuse. A cohort study of Olympic Athletes revealed that 49\% had suboptimal sleep quality. Emerging data suggests a higher rate of suicidal ideation amongst transgender compared to cisgender athletes. Regarding the mental health of para athletes, there is currently limited available data. The International Olympic Committee Sport Mental Health Assessment Tool 1 is a great screening tool that has been psychometrically validated in athletes. It is also important to plan strategic psychopharmacological and psychotherapeutic interventions. The most effective approaches for preventing and managing mental health issues are mindfulness and cognitive-behavioural therapy.

Conclusions Athletes are humans first. Integrating a bio-psycho-social model into the existing healthcare strategy should be a priority. The future of sport should include a mental health action plan for each country. In order for the sport culture to evolve, all those involved should start considering the price of winning a medal.

\section{INJURIES IN LONG DISTANCE RACECAR DRIVERS: A LONGITUDINAL STUDY OF PRE-PARTICIPATION EXAMINATIONS}

Josefine Stoll, Lucie Risch, Michael Cassel, Frank Mayer. University of Potsdam, Outpatient Clinic, Potsdam, Germany

\subsection{6/bjsports-2021-IOC.240}

Background Professional long distance racecar drivers are exposed to high physical load during races. Athletes have to interact with other racecar drivers during high-speed situations on the track. Additionally G-forces have to be counteracted and pedal power maintained without any loss of performance. Data regarding common injuries or overuse are rare and not longitudinal analyzed.

Objective This study is based on prospective longitudinal analyses of common injuries and overuse in professional long distance racecar drivers during pre- participation examinations (PPE).

Design Prospective longitudinal study

Setting University outpatient clinic, Medical examination center of the German Olympic Sports Federation

Patients (or Participants) 37 male professional long distance racecar drivers were analyzed over 15 years (2003-2017).

Interventions (or Assessment of Risk Factors) Athletes participated in a PPE screening two times a year. Frequent diagnoses were evaluated during medical examination focussing on orthopaedics, internal medicine and cardiology. Overall 353 PPEs were included in the analysis of prevalence. Individual follow up of athletes ranged from one to maximum fifteen years.

Main Outcome Measurements The number of findings in medical history and clinical examination was differentiated between orthopaedic, internal medicine and cardiological findings (frequencies [\%]).

Results Long distance racecar drivers presented a low prevalence of injuries. $40 \%$ of cases were found in the musculoskeletal system, $20 \%$ in internal medicine. Frequent complaints were tendinopathies of the lower limb as well as chronic unspecific low back pain. Repetitive infections of the upper respiratory tract and allergies were frequent as well. Cardiological findings were rare.

Conclusions There is a need of regular health monitoring in long distance racecar driving. Prevention of frequent 